\title{
FRACTAL PROPERTIES OF PERIODIC PLASMA WAVEGUIDES
}

\author{
V.I. Lapshyn, G.I. Zaginaylov', V.I. Tkachenko, I.V. Tkachenko \\ NSC KIPT, Kharkov, Ukraine, \\ E-mail tkachenko@kipt.kharkov.ua \\ ${ }^{1}$ V.N. Karazin Kharkov National University, \\ Svobody Sq. 4, Kharkov, 61077, Ukraine
}

Fractal properties of periodic plasma waveguides are investigated in this article. It's shown that the Hausdorff dimension of model dispersion law is $d_{H}=1 / 2$ and for the Trivelpicec-Gould waves $d_{H}=1 / 3$. These values indicate on fractal properties of investigated spectra.

PACS numbers: 52.40.Fd

Recently great interest is attracted by the problems concerning investigation of electromagnetic properties of periodic vacuum systems with plasma filling, so called hybrid structures $[1,2]$. This interest is caused mostly by the possibility of creating of new powerful generators and accelerators due to the amplitude increasing comparatively to those ones but with vacuum filling. Interesting and hopeful results are received in this area [3]. However, "dense" spectra appearance at frequencies below plasma frequency can lead to the stochastic behavior of generated waves.

In present article we will investigate fractal properties of periodic plasma waveguide at the region of frequencies below plasma frequency. Let's consider, for example, two kinds of dispersion law, given by: a model dispersion functions

$$
\omega_{n}=\omega_{p}\left(\frac{k_{n}}{k_{n}+1}\right),
$$

and a dispersion law of Trivelpiece-Gould (TG) waves [4], where $k_{n}=n k_{o}+|k|, \quad n=0, \pm 1, \pm 2 \ldots, \pm N$ amount of harmonics in spectra, $k$ - longitudinal wavenumber.

Let's investigate fractal properties of "dense" spectrum. We will define the Hausdorff dimension of (1) ${ }_{n}$ multiplicity by formula [5]

$$
d_{H}=\lim _{N \rightarrow \infty} \frac{\ln N(r)}{\ln (1 / r)}
$$

Let's consider $k_{o}=1$ and examine distribution of intersection points of dispersion curves with $k=\frac{k_{0}}{2}$ (See, Fig. 1).

In capacity of $N(r)$ and $r$ we will choose $n$ and $\Delta r=\tilde{\omega}_{n}-\widetilde{\omega}_{n-1}$ accordingly, where $\widetilde{\omega}_{n}=\frac{\omega_{n}}{\omega_{p}}$. Then for the Hausdorff dimension we'll obtain $d_{H}=1 / 2$.

Analysis of the Trivelpiece-Gould (TG) waves under the potential limit gives the value of Hausdorff dimension $d_{H}=1 / 3$.
Received values of $d_{H}$ indicate on fractal properties of the investigated spectra. Essentially fractal dimension reflects scale invariance of the investigated multiplicity. I.e. for any frequency close, but less $\omega_{p}$ infinite amount of modes exists by any resolution by wavenumber. This can sufficiently change physics of generation or acceleration in such systems.

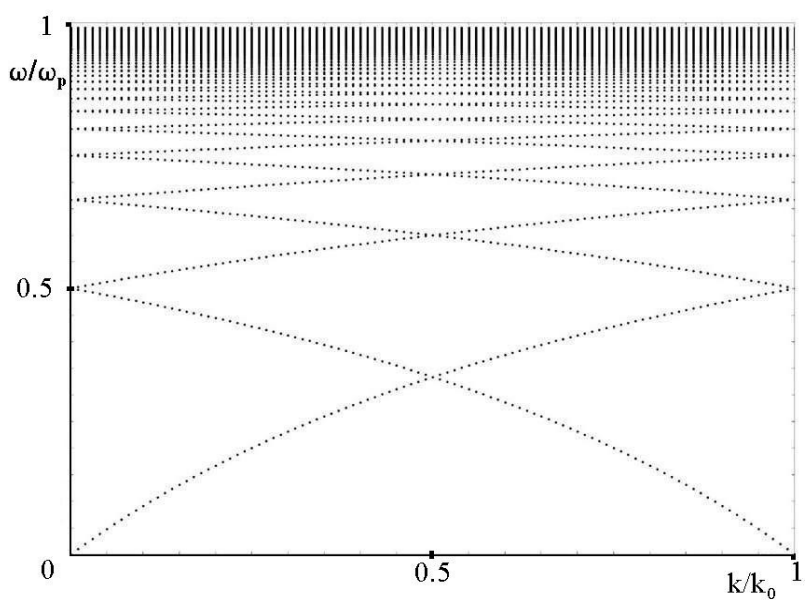

Fig. 1. Fractal distribution of intersection points of dispersion curves with $k=\frac{k_{0}}{2}$.

\section{REFERENCES}

1. V.A.Balakirev, N.I.Karbushev et al. Theory of Cherenkov Accelerators and Generators On Relativistic Beams. Kiev: "Naukova Dumka", 1993, $208 \mathrm{p}$.

2. Ya.B.Fainberg et al. // Doklady AN USSR. 1990, v. A, № 11, p. 55-56.

3. A.A.Antonov et al. // Proc. 10 International Conference on High Power Particle Beams (BEAMS'94) June 20-24, San Diego, 1990, v. 1, p. 260-263.

4. N.Krall, A.Trivelpiece. Principles of plasma physics. Moscow: "Nauka", 1973, $324 \mathrm{p}$.

5. G.M.Zaslavskij, R.Z.Sagdeev. Introduction to the Non-linear Physics. Moscow: "Nauka", 1988, p. 177-186. 